ఠ

\title{
miR-I 24 suppresses proliferation and invasion of nasopharyngeal carcinoma cells through the Wnt/ $\beta$-catenin signaling pathway by targeting Capn4
}

This article was published in the following Dove Press journal:

OncoTargets and Therapy

23 May 2017

Number of times this article has been viewed

\author{
Haili $\mathrm{Hu}^{\mathrm{l}, *}$ \\ Guanghui Wangl,* \\ Congying $\mathrm{Li}^{2}$ \\ 'Department of Otorhinolaryngology, \\ Huaihe Hospital of Henan \\ University, ${ }^{2}$ Department of \\ Otorhinolaryngology, School of \\ Medicine, Kaifeng University, Kaifeng, \\ People's Republic of China \\ *These authors contributed equally \\ to this work
}

Background: Recent studies have demonstrated that microRNA 124 (miR-124) acts as a tumor suppressor in nasopharyngeal carcinoma (NPC); however, the exact molecular mechanism by which miR-124 exerts tumor suppression has not been well elucidated.

Materials and methods: We performed quantitative real-time PCR (qRT-PCR) to measure the expression of metastasis associated lung adenocarcinoma transcript 1, miR-124, and calpain small subunit 1 (Capn4) mRNAs in NPC cell lines. We also performed western blot analysis to detect the levels of Capn4. Furthermore, we performed MTT assay and transwell invasion assay to determine the proliferation and invasion ability of two NPC cell lines, namely, HONE1 and CNE2 cells, respectively. The verification of targets of miR-124 was performed using prediction softwares and luciferase reporter analysis.

Results: According to our results, the expression of Capn4 was found to be elevated, whereas the expression of miR-124 was lowered in NPC cell lines compared with normal nasopharyngeal cells. When we preformed overexpression of miR-124, it suppressed the proliferation and invasion of NPC cells. Moreover, miR-124 suppressed the expression of Capn 4 by targeting Capn4 in HONE1 and CNE2 cells. When we preformed overexpression of Capn4, it reversed the inhibitory effect of miR-124 on the proliferation and invasion of NPC cells. Furthermore, miR-124-Capn 4 axis decreased the levels of $\beta$-catenin, cyclin D1, and c-Myc, the components of the Wnt/ $\beta$-catenin signaling pathway.

Conclusion: The suppression of proliferation and invasion of NPC cells by miR-124 were achieved by the regulation of Wnt/ $\beta$-catenin signaling pathway by targeting Capn 4 . The results of this study revealed a novel miR-124-Capn4 regulatory axis in NPC cell lines, providing a better understanding of the pathogenesis of NPC and a promising therapeutic target for patients with NPC.

Keywords: miR-124, Calpain small subunit 1, NPC

\section{Introduction}

Nasopharyngeal carcinoma (NPC) is an epithelial malignancy of the uppermost portion of the pharynx, characterized by high invasion and metastasis. ${ }^{1}$ The prevalence of NPC is highest in distinct endemic regions such as Southeast Asia, where the annual occurrence can reach up to $1 / 4,000 .^{2}$ The long-term survival of patients with NPC is usually excellent when detected at an early stage; however, patients with locally advanced and metastatic NPC showed poor prognosis. ${ }^{3}$ Like many other cancers, multiple genetic and epigenetic aberrancies could lead to the tumorigenesis and progression of NPC. ${ }^{4}$ However, the precise mechanism underlying the progression of NPC remains to be elucidated.

MicroRNAs (miRNAs) are endogenous small non-coding RNA molecules with about 20 nucleotides in length that regulate the expression of their target genes through
Correspondence: Haili Hu Department of Otorhinolaryngology, Huaihe Hospital of Henan University, I 15 Ximen Street, Kaifeng 475200,

People's Republic of China

Tel +86 37l 2390659 I

Email huhaili12@I63.com (c)
hereby accept the Terms. Non-commercial uses of the work are permitted without any further permission from Dove Medical Press Limited, provided the work is properly attributed. For permission hereby accept the Terms. Non-commercial uses of the work are permitted without any further permission from Dove Medid 
mRNA degradation or translational inhibition. ${ }^{5}$ Accumulating evidence suggests that miRNAs function either as tumor suppressors or as oncogenes, regulating tumor initiation and progression at various levels. ${ }^{6}$ To date, numerous miRNAs have been reported to be dysregulated in NPC, such as miR-144, miR-214, and miR-10b, contributing to the development of NPC and its progression. ${ }^{7-9}$ It is noteworthy that miR-124 generally acts as a tumor suppressor in multiple cancers, such as breast cancer, ${ }^{10}$ colorectal carcinoma, ${ }^{11}$ and NPC. ${ }^{12}$ Moreover, downregulation of miR-124 has been reported in NPC, and restoration of expression of miR-124 suppressed the proliferation, migration, and invasion of NPC cells. ${ }^{12,13}$ However, the mechanism by which miR-124 regulates NPC progression needs to be further investigated.

Calpains represent a family of calcium-dependent neutral cysteine proteases. ${ }^{14}$ Many studies have demonstrated the importance of aberrant calpain expression during tumorigenesis. ${ }^{15}$ Calpain small subunit 1 (Capn4) is a small regulatory subunit of the Calpain family and plays a crucial role in the maintenance of calpain's stability and activity. ${ }^{15}$ A previous study indicated that the overexpression of Capn4 was the underlying cause of invasion and metastasis after liver transplantation in hepatocellular carcinoma. ${ }^{16}$ Another study revealed that Capn4 was upregulated and was associated with tumor progression and its clinical outcome in clear cell renal cell carcinoma. ${ }^{17}$ Another study revealed that Capn4 was highly expressed in NPC cell lines and that the knockdown of Capn 4 caused the suppression of cell migration and invasion both in vitro and in vivo. ${ }^{18}$ However, the regulatory mechanism of Capn4 in NPC is still unknown.

In this study, we first explored whether miR-124 regulates the tumor progression in NPC. Furthermore, we investigated the underlying mechanism by which miR-124 exerts its function in NPC.

\section{Materials and methods}

\section{Cell culture}

The human NPC cell lines (HONE1, CNE1, and CNE2), nasal epithelial cell line (HNEpC), and immortalized nasopharyngeal epithelial cell line (NP69) were purchased from the American Type Culture Collection (ATCC). All cells were maintained in Roswell Park Memorial Institute (RPMI)-1640 (Thermo Fisher Scientific, Carlsbad, CA, USA) with 10\% fetal bovine serum (FBS; Thermo Fisher Scientific) and cultured at $37^{\circ} \mathrm{C}$ in a humidified atmosphere with $5 \% \mathrm{CO}_{2}$.

\section{Quantitative real-time PCR ( $q$ RT-PCR)}

Total RNAs were isolated from HNEpC, NP69, and NPC cell lines using Trizol (Thermo Fisher Scientific). The first-strand
cDNA of Capn4 was synthesized by using a high capacity cDNA reverse transcription kit (Applied Biosystems, Foster City, CA, USA). A TaqMan MicroRNA Reverse Transcription kit (Applied Biosystems) was used to generate cDNA of miR-124. We performed qRT-PCR by using All-in-One ${ }^{\mathrm{TM}}$ qPCR Mix (GeneCopoeia, Rockville, MD, USA) on an ABI 7500HT System (Applied Biosystems). $\beta$-Actin and U6 were used as endogenous controls for Capn4 and miR-124, respectively. The primers used in the protocol were as follows: miR-124, 5'-ACACTCCAGCTGGGTAAGGCACGCGGTG-3' (forward) and 5'-TGGTGTCGTGGAGTCG-3' (reverse); U6, 5'-CTCGCTTCGGCAGCACA-3' (forward) and 5'-TGGTGTCGTGGAGTCG-3' (reverse); Capn4, 5'-ACC CACTCCGTAACCTC-3' (forward) and 5'-GGGTAGC AACCGTGAA-3' (reverse); and $\beta$-actin, $5^{\prime}$-TGAGAGGGAA ATCGTGCGTGAC-3' (forward) and 5'-AAGAAGGAAGG CTGGAAAAGAG-3' (reverse). The relative levels of RNAs were analyzed using the $2^{-\Delta \Delta \mathrm{Ct}}$ method.

\section{Western blot analysis}

We performed western blot analysis as previously described. ${ }^{19}$ The anti-Capn4 antibody was purchased from LifeSpan Biosciences (Seattle, WA, USA). The anti- $\beta$-catenin antibody was obtained from BD Bioscience (San Jose, CA, USA). Anti-cyclin D1, anti-c-Myc, and anti- $\beta$-actin antibodies were products of Santa Cruz Biotechnology (Santa Cruz, CA, USA). Protein bands were detected with enhanced chemiluminescence reagent (Thermo Scientific, Logan, UT, USA) and visualized by a chemiluminescence detector (BioRad Laboratories, Hercules, CA, USA). The densitometric analysis of blots was performed by using Bio-Rad Image Lab 4.0 software (Bio-Rad Laboratories).

\section{Cell transfection}

The pcDNA-Capn4 and pcDNA-control were purchased from Thermo Fisher Scientific. We purchased miR-124 mimics, anti-miR-124, and miR-control from GenePharma (Shanghai, China). Cell transfection was performed using Lipofectamine 2000 (Thermo Fisher Scientific).

\section{Cell proliferation assay}

The cell viability of HONE1 and CNE2 was examined using the MTT assay, which was performed after transfection for 24,48 , and $72 \mathrm{~h}$. The absorbance at $450 \mathrm{~nm}$ was determined after incubation with $20 \mu \mathrm{L}$ of MTT (Sigma, St Louis, MO, USA) for $4 \mathrm{~h}$ using a microtiter plate reader (Molecular Devices, Sunnyvale, CA, USA). 


\section{Cell invasion assay}

The invasion ability of HONE1 and CNE2 cells was determined by transwell invasion assay. Cells incubated in serumfree medium were placed into the upper chamber pre-coated with Matrigel (BD Bioscience) and the cell culture media containing $10 \%$ FBS was added into the lower chamber. After $48 \mathrm{~h}$, the invaded cells were fixed, stained with $0.5 \%$ crystal violet solution, and counted under a microscope (Olympus, Japan).

\section{Luciferase reporter assays}

Wild-type or mutant 3'UTR fragments (3'UTR-WT or $3^{\prime}$ UTR-MUT) of Capn4 were amplified and then cloned into the luciferase reporter vectors pGL3 (Ambion, Applied Biosystems, Foster City, CA, USA). Subsequently, HONE1 and CNE2 cells were co-transfected with miR-control or miR-124 and wild- or mutant-type reporter plasmids (WT or MUT). Luciferase activities were measured using the Dual-Luciferase Reporter Assay System (Promega, Madison, WI, USA) $48 \mathrm{~h}$ post-transfection. Renilla luciferase was used as an endogenous reference for normalization of luciferase activity.

\section{A}

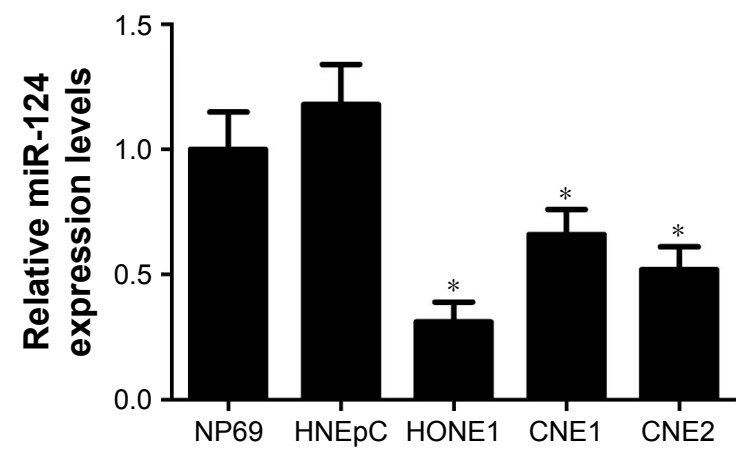

C

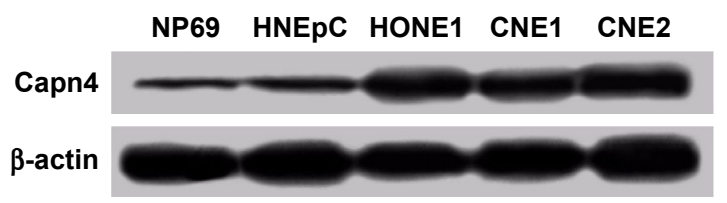

\section{Statistical analysis}

All experiments were repeated for at least three independent times. Statistical analyses were performed using SPSS 19.0 software (SPSS, Chicago, IL, USA). The statistical differences were determined using Student's $t$-test or one-way analysis of variance. Differences were considered to be statistically significant when $P<0.05$.

\section{Results}

\section{Decreased expression of miR-I24 and increased expression of Capn4 in NPC cell lines}

The expression of miR-124 and Capn 4 mRNA was detected by qRT-PCR analysis, and Capn 4 protein level was measured using western blot in HNEpC, NP69, and NPC cell lines. According to the results of qRT-PCR analysis, miR-124 expression was enormously reduced in NPC cell lines compared with HNEpC or NP69 cells (Figure 1A). In contrast, the levels of Capn 4 mRNA (Figure 1B) and protein (Figure 1C) were evidently upregulated in NPC cell lines. These data demonstrated that dysregulated miR-124 and Capn 4 expression may be involved in the pathogenesis of NPC.
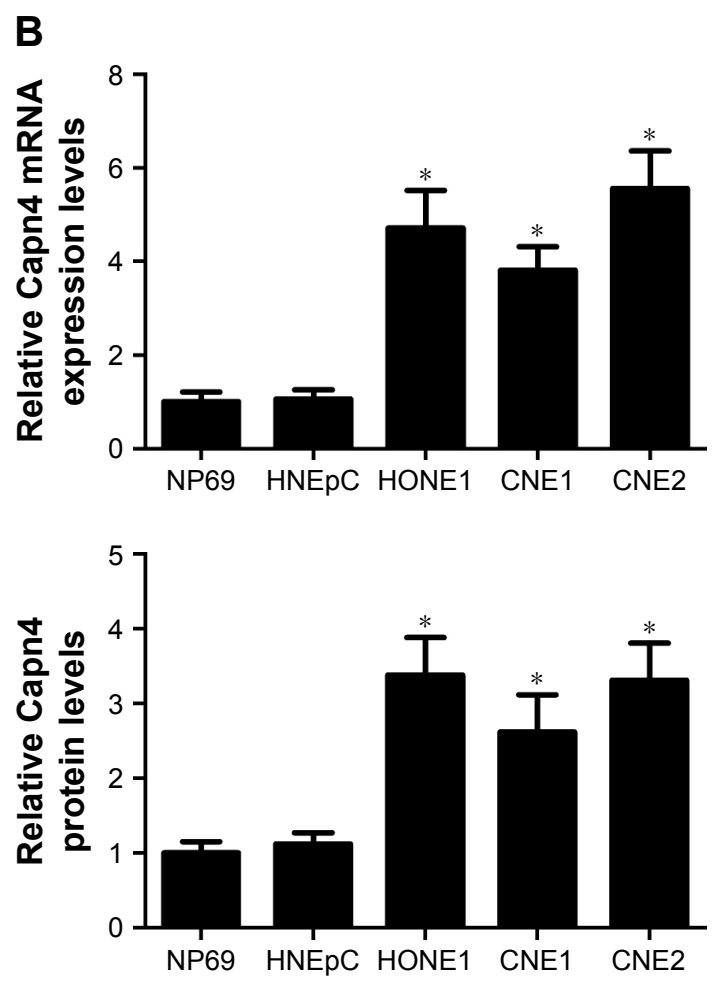

Figure I The level of microRNA-I24 (miR-124) was downregulated and Capn4 expression was upregulated in NPC cell lines (HONEI, CNEI, and CNE2) compared with human nasal epithelial cell line (HNEpC) or immortalized nasopharyngeal epithelial cell line (NP69).

Notes: (A) Quantitative real-time PCR (qRT-PCR) analysis revealed decreased miR-124 expression in HONEI, CNEI, and CNE2 cells. (B) qRT-PCR analysis indicated that the expression level of Capn4 mRNA was elevated in HONEI, CNEI, and CNE2 cells. (C) Western blot analysis showed the increased protein level of Capn4 in HONEI, CNEI, and CNE2 cells. Data are shown as mean \pm standard deviation $(n=3)$. $* P<0.05$ NPC cells vs HNEpC or NP69. 


\section{Overexpression of miR-I 24 inhibits} proliferation and invasion of NPC cells

To investigate the effect of miR-124 on NPC cells, miR-124overexpressing HONE1 and CNE2 cells were constructed by miR-124 mimic transfection. The cell viability of NPC cells was determined by MTT assay. According to the results, the restored miR-124 expression led to a significant reduction in HONE1 and CNE2 cell viabilities compared with that of the control groups (Figure 2A). Subsequently, transwell invasion assay was performed to assess the effect of miR-124 on NPC cell invasion. Compared with the control groups, the cell invasion capacity of HONE1 and CNE2 cells was significantly suppressed by enforced expression of miR-124 (Figure 2B). Collectively, these results illustrate that miR-124 suppresses proliferation and invasion of NPC cells.
Capn4 is a functional target of miR- 124

Accumulating evidence suggested that miRNAs exert their function by suppressing special mRNA targets. Therefore, online TargetScan software was used to predict the target genes of miR-124. As shown in Figure 3A, Capn4 was a predicted binding site of miR-124. Then dual luciferase reporter assay was performed to confirm the miR-124 target. The luciferase activity of Capn4-WT reporter in HONE1 and CNE2 cells was obviously lowered by miR-124; however, the luciferase activity of Capn4-MUT reporter had no significant change after treatment (Figure 3B). To further confirm the effect of miR-124 on the expression of Capn4, western blot analysis was performed to detect the protein levels of Capn 4 in miR-124 or anti-miR-124 transfected cells. The overexpression of miR-124 resulted in a significant suppression of
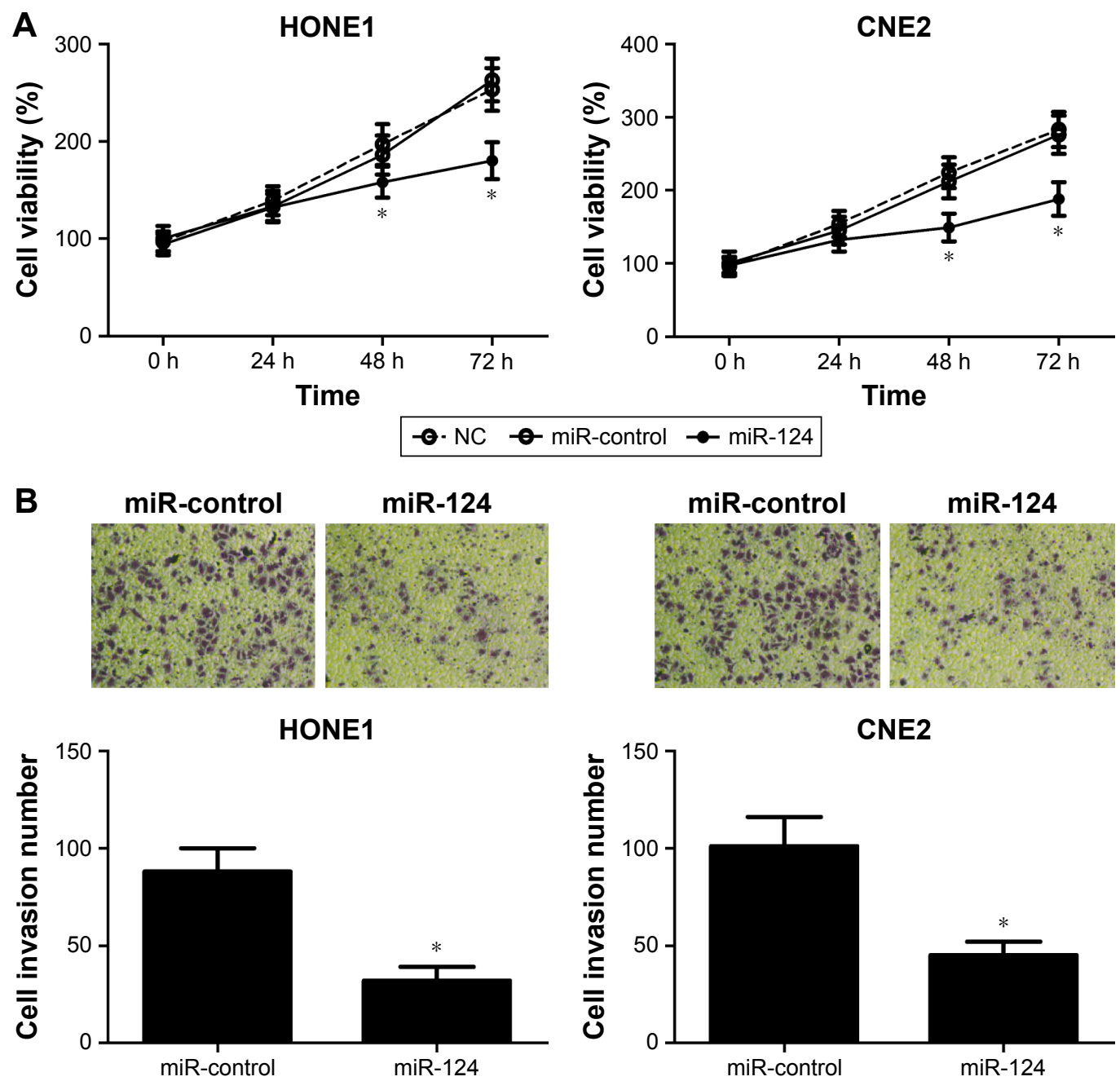

Time
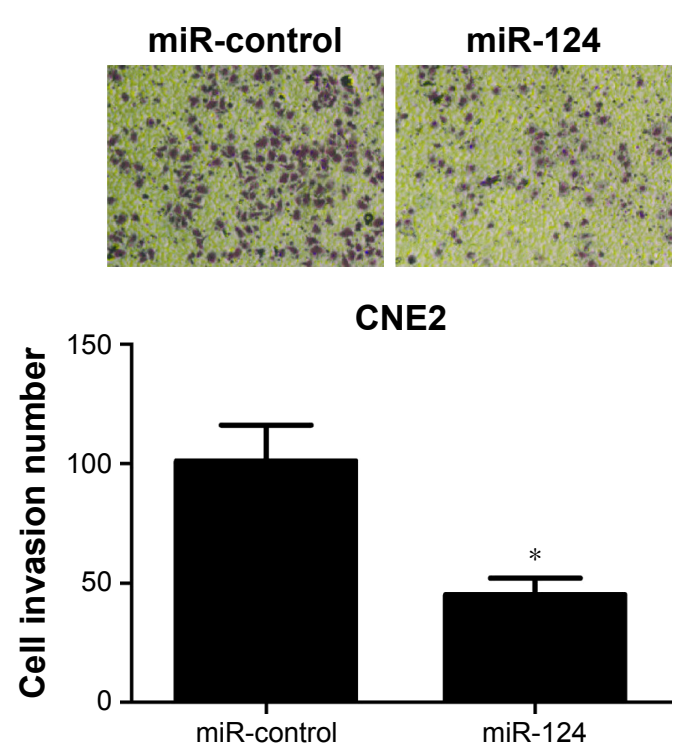

Figure 2 MicroRNA-I24 (miR-124) inhibited proliferation and invasion of nasopharyngeal carcinoma (NPC) cell lines HONEI and CNE2. HONEI and CNE2 cells were transfected with miR-control or miR-124.

Notes: (A) MTT assay revealed that the cell viability decreased 24, 48, and $72 \mathrm{~h}$ after miR- 124 transfection in HONEI and CNE2 cells when compared with the miR-control or untreated cells (NC group). Data are shown as mean \pm standard error of mean $(n=3)$. $* P<0.05$ miR- 124 treated cells vs miR-control treated cells or untreated cells. (B) Transwell invasion assay showed that the invasion of HONEI and CNE2 cells was suppressed after transfection with miR-I24. Data are shown as mean \pm standard deviation ( $\mathrm{n}=3$ ). $* \mathrm{P}<0.05$ miR- 124 treated cells vs miR-control treated cells.

Abbreviation: NC, untreated cells. 


\section{A Capn4 3'UTR-WT 5'... GACCCAGgugCCCCAGUGCCUUU... \\ miR-124 3'... CCGUAAGUGGCGCACGGAAU \\ Capn4 3'UTR-MUT 5'...GACCCAGGUGCCCCA ACUAUCAU...}
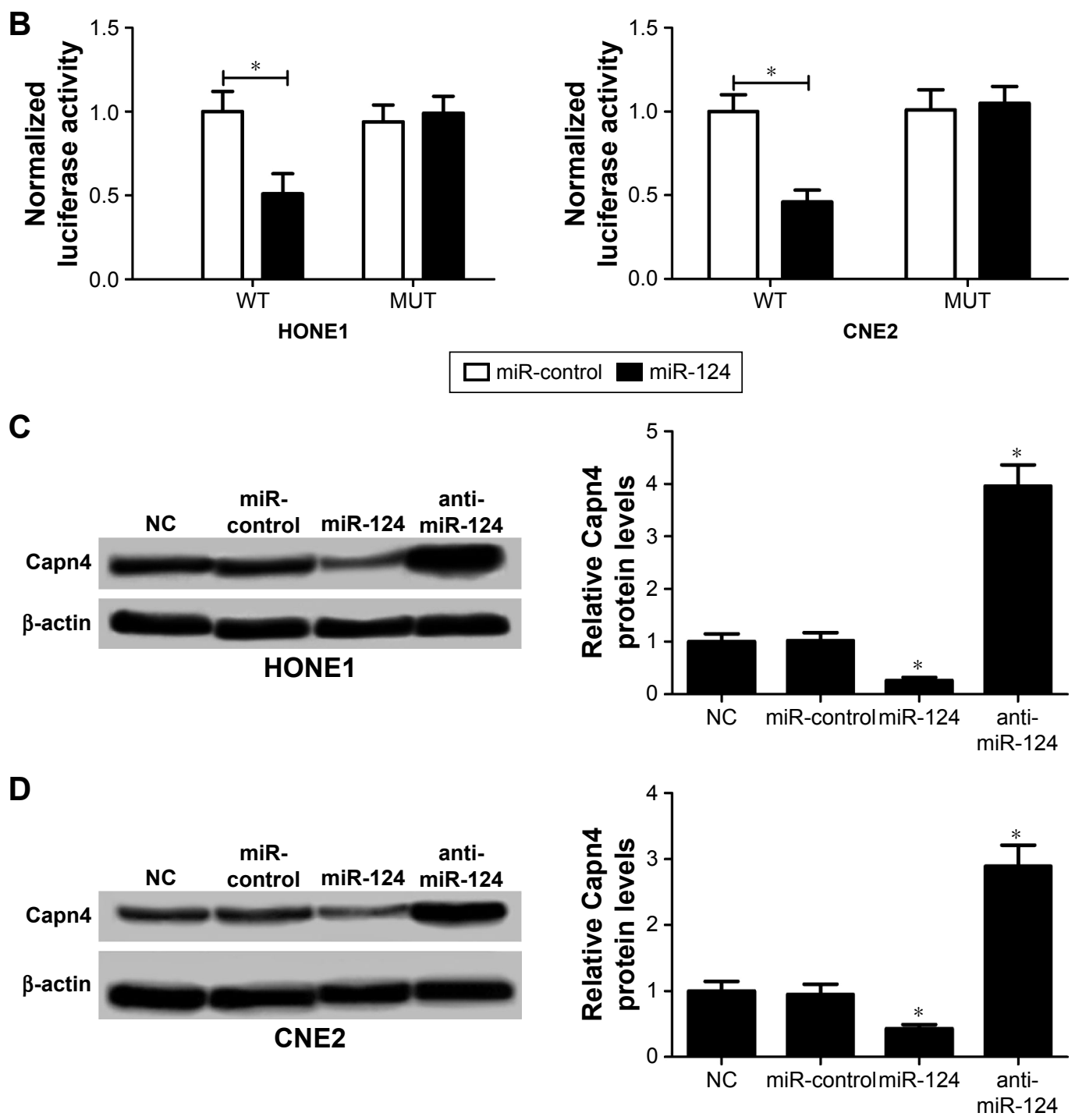

Figure 3 MicroRNA-I24 (miR-124) directly suppressed Calpain small subunit I (Capn4) expression.

Notes: (A) Putative miR-124 binding sequence of Capn4 was shown. (B) The relative luciferase activity was detected in HONEI and CNE2 cells co-transfected with wildtype or mutant Capn4 3'UTR and miR-I 24 mimic or miR-control. $* P<0.05$ (C and D) Western blot analysis indicated that the level of Capn4 expression was decreased by miR-I 24 transfection and elevated by miR- 124 inhibition in HONEI and CNE2 cells. NC represents untreated cells. Data are shown as mean \pm standard deviation ( $\mathrm{n}=3$ ). $* P<0.05$ miR- 124 treated cells vs miR-control treated cells.

Abbreviations: MUT, mutant; NC, untreated cells; WT, wild-type.

Capn4 expression, whereas miR-124 inhibition markedly led to the improvement of Capn4 levels in HONE1 and CNE2 cells (Figure 3C and D). Overall, these data implied that Capn4 is a functional target of miR-124.

\section{Capn4 upregulation reverses the effects of miR-I 24 overexpression on proliferation and invasion of NPC cells}

To further confirm the underlying mechanism of miR-124 in NPC cell proliferation and invasion, HONE1 and CNE2 cells were transfected with miR-124 or co-transfected with miR-124 and pcDNA-Capn4. MTT assay revealed that Capn4 overexpression reversed the inhibitory effect of miR-124 on cell proliferation of HONE1 and CNE2 (Figure 4A). In addition, transwell invasion assay revealed that transfection of pcDNA-Capn4 overturned the inhibited invasion of HONE1 (Figure 4B) and CNE2 (Figure 4C) cells caused by the overexpression of miR-124. All these data demonstrated that miR-124 suppressed proliferation and invasion of NPC cells by the targeted inhibition of expression of Capn4. 
A

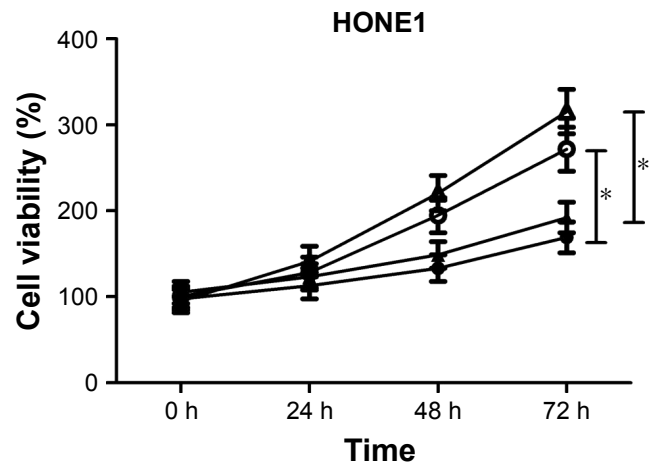

CNE2

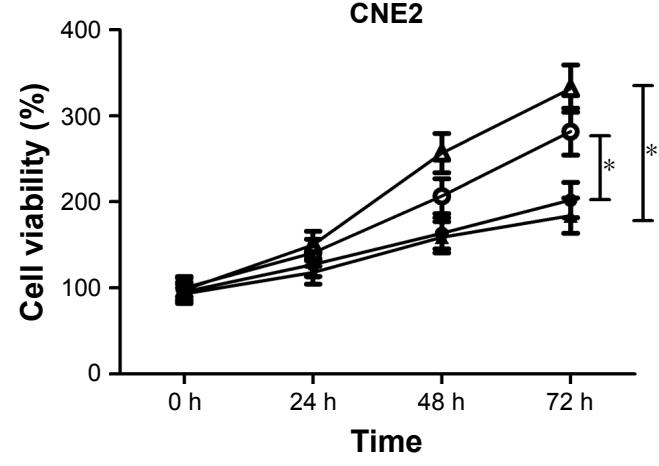

- miR-control $\rightarrow$ miR-124 $\leftarrow$ miR-124 + vector $₫$ miR-124 + pcDNA-Capn4

B

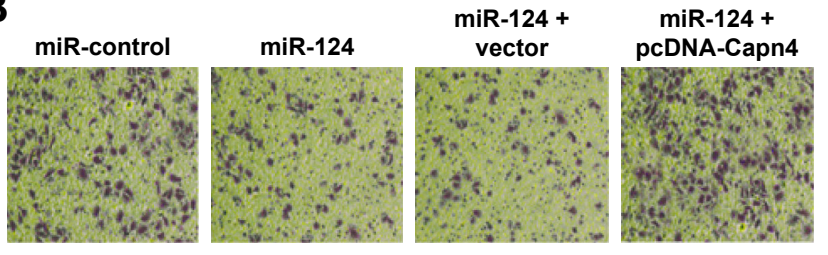

HONE1

C

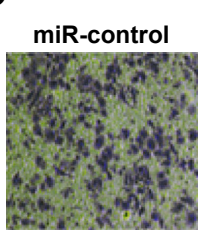

miR-124

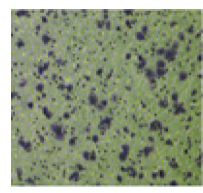

miR-124 + vector

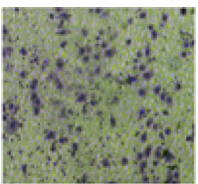

CNE2
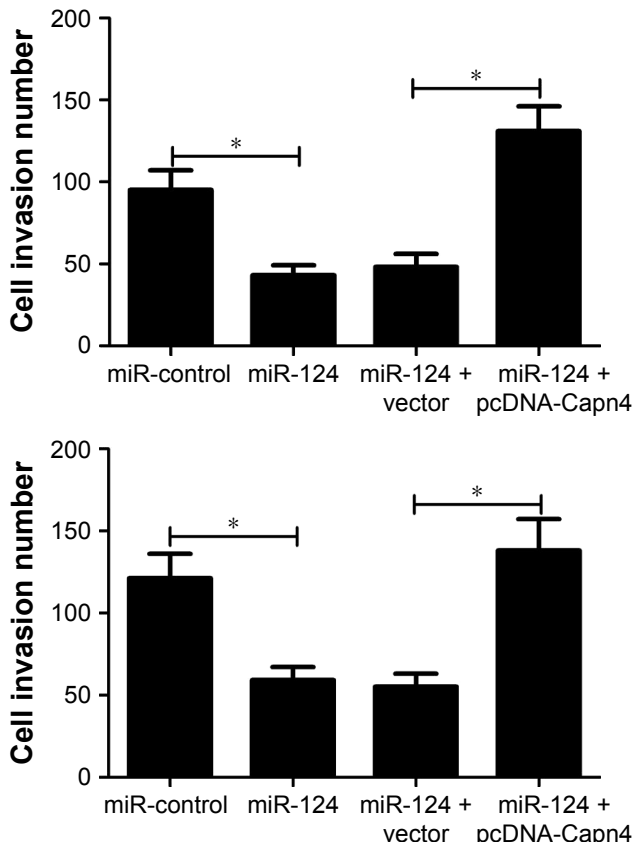

Figure 4 MicroRNA-I24 (miR-124) suppressed proliferation and invasion of NPC cells through the inhibition of Capn4 expression. HONEI and CNE2 cells were transfected with miR-I 24 or in combination with pcDNA-Capn4.

Notes: (A) MTT assay confirmed that restored Capn4 expression reversed the inhibitory effect of miR-124 on the cell viability of HONEI and CNE2 cells at 24 , 48 , and $72 \mathrm{~h}$ after transfection. Data are shown as mean \pm standard error of mean $(\mathrm{n}=3)(\mathbf{B}$ and $\mathbf{C})$ Transwell invasion assay suggested that transfection of pcDNA-Capn 4 abolished miR-I24-mediated inhibition of cell invasion in HONEI and CNE2 cells. Data are shown as mean \pm standard deviation $(n=3)$. $* P<0.05$.

Abbreviation: NPC, nasopharyngeal carcinoma.

\section{Wnt/ $\beta$-catenin signaling pathway inhibition suppresses proliferation and invasion of NPC cells}

To investigate the role of $\mathrm{Wnt} / \beta$-catenin signaling pathway in NPC cells, the cell viability and invasion ability of HONE1 and CNE2 cells treated with different doses of XAV939 $(0,10$, and $20 \mu \mathrm{M})$, a $\mathrm{Wnt} / \beta$-catenin signaling pathway inhibitor, was determined. MTT assay revealed that XAV939 inhibited the proliferation of HONE1 and CNE2 cells in a dose-dependent manner (Figure 5A). In addition, the results of transwell invasion assay indicated that the invasion ability of HONE1 and CNE2 cells was suppressed by XAV939 in a dose-dependent manner (Figure 5B). To sum up, inhibition of the Wnt $/ \beta$-catenin signaling pathway contributed to the suppression of proliferation and invasion of NPC cells.

\section{The miR-I24-Capn4 axis inhibits the $W n t / \beta$-catenin signaling pathway}

To further elucidate whether miR-124 or Capn 4 affected the proliferation and invasion of NPC cells through the regulation of Wnt/ $\beta$-catenin signaling pathway, $\beta$-catenin, cyclin D1, and c-Myc protein levels were examined in HONE1 and CNE2 cells transfected with miR-124 or co-transfected with miR-124 and pcDNA-Capn4. Western blot analysis revealed that the upregulation of miR-124 led to a decrease in $\beta$-catenin, cyclin D1, and c-Myc expression in HONE1 and 

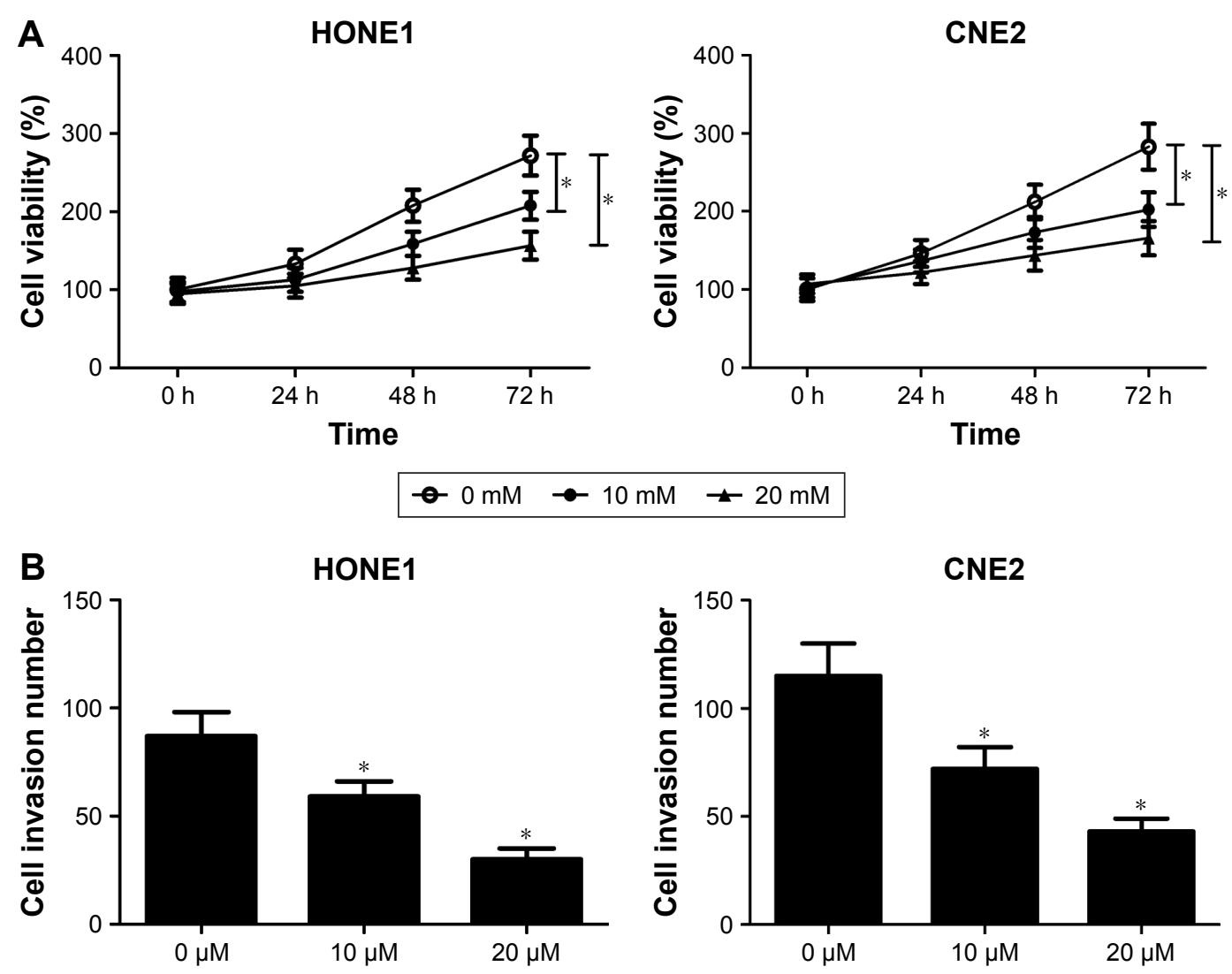

Figure 5 The Wnt/ $\beta$-catenin signaling pathway inhibitor, XAV939, suppressed proliferation and invasion of nasopharyngeal carcinoma (NPC) cells. HONEI and CNE2 cells were treated with different doses of $\operatorname{XAV} 939(0,10$, and $20 \mu \mathrm{M})$.

Notes: (A) MTT assay displayed that the cell viability of HONEI and CNE2 cells was inhibited by XAV939 in a dose-dependent manner at 24,48 , and $72 \mathrm{~h}$ after XAV939 treatment. Data are shown as mean \pm standard error of mean $(n=3) * P<0.05$. (B) Transwell invasion assay indicated that the invasion ability of HONEI and CNE2 cells was inhibited by XAV939 in a dose-dependent manner after treatment with XAV939 for $48 \mathrm{~h}$. Data are shown as mean \pm standard deviation ( $\mathrm{n}=3$ ). $* \mathrm{P}<0.05 \mathrm{XAV} 939$ treated cells vs control media treated cells.

CNE2 cells; however, ectopic expression of Capn4 abolished the inhibitory effect of miR-124 on these protein expressions (Figure 6A and B). Collectively, miR-124 suppressed the Wnt/ $\beta$-catenin signaling pathway through the inhibition of Capn4 expression.

\section{Discussion}

Recently, mounting evidence suggests that miRNAs function either as tumor suppressors or as oncogenes involved in prevalent cancers. Among these miRNAs, miR-124 is generally expressed lower in several cancers and functions as a tumor suppressor. For instance, the downregulation of miR-124 led to an increased cell proliferation and cell cycle progression in breast cancer. ${ }^{10}$ Moreover, miR-124 was expressed low in tumor tissues when compared with the normal tissues, and exogenetic expression of miR-124 led to a reduced cell proliferation, delayed colony formation, and decreased tumor growth in head and neck squamous cell carcinomas by directly inhibiting SphK1 activity and its downstream signals. ${ }^{20}$ In breast cancer, miR-124 was noticeably downregulated, and restored miR-124 expression attenuated cell viability, proliferation, and cell cycle progression through targeting cyclin-dependent kinase- $4 .{ }^{21}$ In this study, we also found that the expression of miR-124 was downregulated in NPC cell lines. In addition, the overexpression of miR-124 resulted in the inhibition of cell proliferation and invasion in NPC cells. In agreement with our findings, previous studies revealed that overexpression of miR-124 dramatically suppressed cell proliferation, colony formation, migration, and invasion of NPC by targeting STAT3 or Foxq1. ${ }^{12,13}$ All these findings suggest that miR-124 functions as a tumor suppressor in NPC.

Capn4 is a small regulatory subunit of the calpain family that regulates a wide spectrum of biological functions. Capn 4 was reported to regulate cell proliferation and differentiation in osteoblasts..$^{22}$ Moreover, Capn4 participates in the DNA damage response and cell cycle modulation. ${ }^{23}$ Capn 4 also plays a key role in tumor migration or invasion. For instance, the downregulation of Capn 4 mediated by miR124 inhibited the invasion and migration of glioma cells. ${ }^{24}$ Capn4 enhanced the invasion ability of non-small cell lung cancer cells by upregulating the expression of matrix metalloproteinase $2 .{ }^{25}$ Capn 4 was highly expressed in NPC cell 

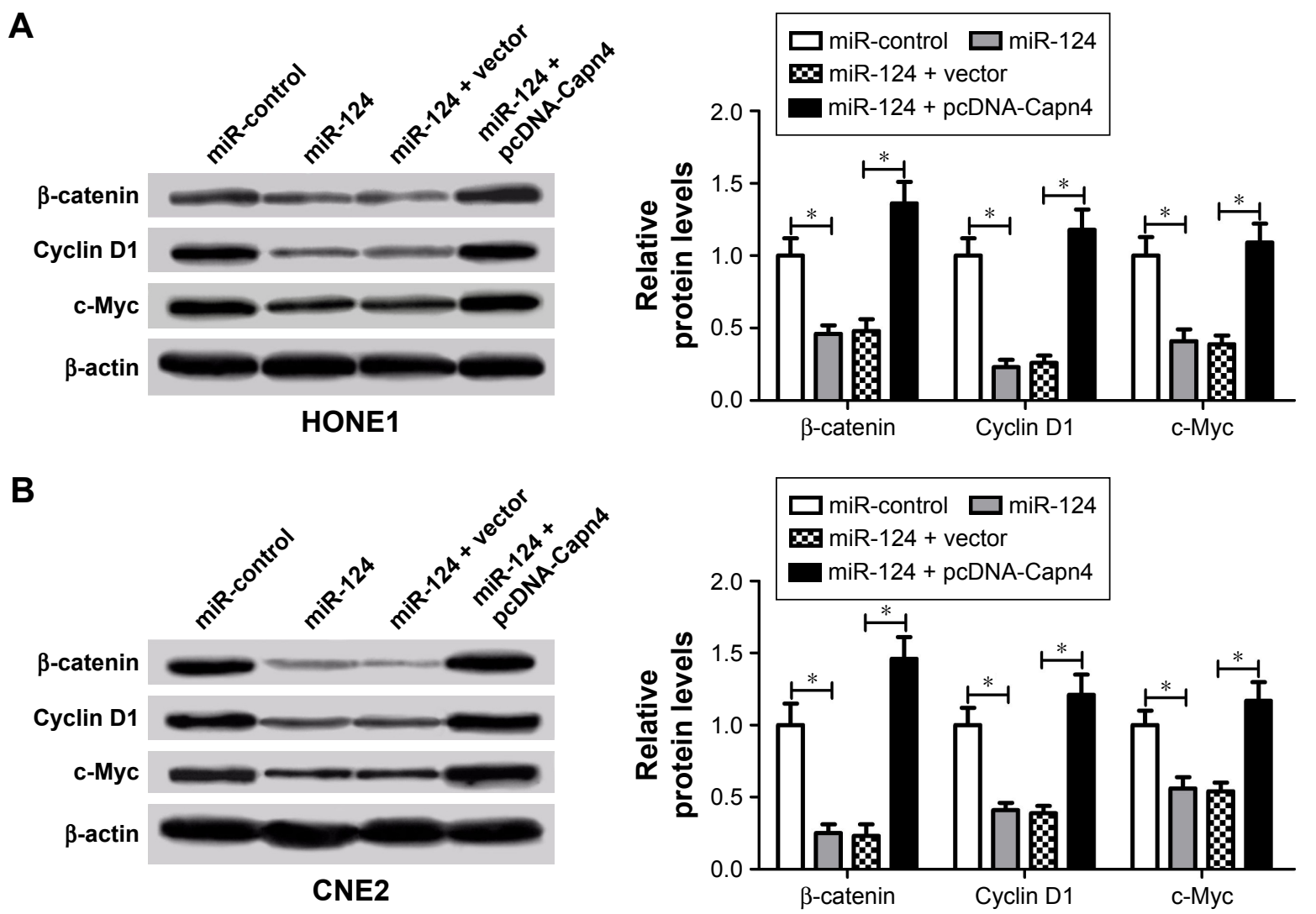

Figure 6 Overexpression of calpain small subunit I (Capn4) reversed the inhibitory effect of miR-I 24 on the Wnt/ $\beta$-catenin signaling pathway. HONEI and CNE2 cells were transfected with miR-I24 or co-transfected with miR-I24 and pcDNA-Capn4 (A and B). Western blot analysis revealed that Capn4 overexpression reversed the decreased $\beta$-catenin, cyclin DI, and c-Myc protein levels caused by the overexpression of miR-I24 in HONEI and CNE2 cells. Data are shown as mean \pm standard deviation ( $\mathrm{n}=3$ ). $* p<0.05$ miR-1 24 or pcDNA -Capn4 treated cells vs miR-control or vector treated cells.

lines, and Capn4 knockdown suppressed in vitro and in vivo cell migration and invasion. ${ }^{18}$ However, the regulation of Capn4 in NPC is still unknown. In this study, Canp4 was verified to be a target of miR-124 by dual luciferase reporter and western blot analyses. Moreover, the overexpression of Capn4 reversed the inhibitory effect of miR-124 on the cell proliferation and invasion in NPC cells. In consistent with our findings, Cai et $\mathrm{al}^{26}$ revealed that miR-124 suppressed the migration and invasion of glioma cells by targeting Capn4. Previous studies also have demonstrated that miR-124 inhibited the proliferation and invasion of NPC cells by modulating its functional targets, such as STAT3 and Foxq1. ${ }^{12,13}$ All these findings suggest that miR-124 regulates different targets in NPC. Therefore, future investigations should focus on elucidating the precise mechanism by which miR-124 plays its roles in NPC.

Accumulating evidence suggests that the Wnt/ $\beta$-catenin signaling pathway regulates several biological responses including proliferation, differentiation, and invasion. ${ }^{27-29}$ For example, activation of the $\mathrm{Wnt} / \beta$-catenin signaling pathway by Wnt3a enhanced the migration and invasion capacity of mesenchymal stem cells. ${ }^{30}$ Moreover, aberrant Wnt/ $\beta$-catenin signaling activities promoted tumor progression in NPC through the regulation of stemness of NPC cells as well as epithelial-mesenchymal transitional events. ${ }^{31,32}$ Recently, Wang et $\mathrm{al}^{33}$ revealed that inactivating the $\mathrm{Wnt} / \beta$-catenin pathway mediated by ZNRF3 inhibited the invasion and tumorigenesis in NPC cells. These results are in good agreement with our findings that inhibition of the Wnt/ $\beta$-catenin signaling pathway inhibited proliferation and invasion of NPC cells. Furthermore, we found that miR-124 could suppress the $\mathrm{Wnt} / \beta$-catenin signaling pathway; however, overexpression of Capn4 reversed the inhibitory effect caused by miR-124. A previous study revealed that miR-124 could downregulate the $\mathrm{Wnt} / \beta$-catenin signaling pathway in rat mesenchymal stem cells. ${ }^{34}$ Another study reported that Capn4 enhanced epithelial-mesenchymal transition in human melanoma cells by the activation of the Wnt/ $\beta$-catenin signaling pathway. ${ }^{35}$ Our results agree with both of these studies. Thus, our findings demonstrated that miR-124 led to inactivity of the Wnt/ $\beta$-catenin signaling pathway by targeting Capn4 in NPC progression. 


\section{Conclusion}

In summary, our study demonstrates that overexpression of miR-124 suppresses proliferation and invasion of NPC cells. More importantly, we provided evidence that miR-124 exerts its functional role via Wnt/ $\beta$-catenin signaling pathway by inhibiting Capn4 in NPC. Altogether, these data suggest that manipulation of the level of miR-124 may provide a novel therapy for NPC patients in the future.

\section{Disclosure}

The authors report no conflicts of interest in this work.

\section{References}

1. Lee KT, Tan JK, Lam AK, Gan SY. MicroRNAs serving as potential biomarkers and therapeutic targets in nasopharyngeal carcinoma: a critical review. Crit Rev Oncol Hematol. 2016;103:1-9.

2. Chang ET, Adami HO. The enigmatic epidemiology of nasopharyngeal carcinoma. Cancer Epidemiol Biomarkers Prev. 2006;15(10): 1765-1777.

3. Chan KC, Chan LS, Ip JC, et al. Therapeutic targeting of CBP/ $\beta$-catenin signaling reduces cancer stem-like population and synergistically suppresses growth of EBV-positive nasopharyngeal carcinoma cells with cisplatin. Sci Rep. 2015;5:9979.

4. Chou J, Lin YC, Kim J, et al. Nasopharyngeal carcinoma - review of the molecular mechanisms of tumorigenesis. Head Neck. 2008; 30(7):946-963.

5. He L, Hannon GJ. MicroRNAs: small RNAs with a big role in gene regulation. Nat Rev Genet. 2004;5(7):522-531.

6. Borchert GM, Lanier W, Davidson BL. RNA polymerase III transcribes human microRNAs. Nat Struct Mol Biol. 2006;13(12):1097-1101.

7. Zhang LY, Ho-Fun Lee V, Wong AM, et al. MicroRNA-144 promotes cell proliferation, migration and invasion in nasopharyngeal carcinoma through repression of PTEN. Carcinogenesis. 2013;34(2):454-463.

8. Deng M, Ye Q, Qin Z, et al. miR-214 promotes tumorigenesis by targeting lactotransferrin in nasopharyngeal carcinoma. Tumor Biol. 2013; 34(3):1793-1800.

9. Sun XJ, Liu H, Zhang P, Zhang XD, Jiang ZW, Jiang CC. miR-10b promotes migration and invasion in nasopharyngeal carcinoma cells. Asian Pac J Cancer Prev. 2013;14(9):5533-5537.

10. Feng T, Shao F, Wu Q, et al. miR-124 downregulation leads to breast cancer progression via LncRNA-MALAT1 regulation and CDK4/E2F1 signal activation. Oncotarget. 2016;7(13):16205-16216.

11. Zhou L, Xu Z, Ren X, Chen K, Xin S. MicroRNA-124 (MiR-124) inhibits cell proliferation, metastasis and invasion in colorectal cancer by downregulating rho-associated protein kinase 1 (ROCK1). Cell Physiol Biochem. 2016;38(5):1785-1795.

12. Peng XH, Huang HR, Lu J, et al. MiR-124 suppresses tumor growth and metastasis by targeting Foxq1 in nasopharyngeal carcinoma. Mol Cancer. 2014;13(1):186.

13. Xu S, Zhao N, Hui L, Song M, Miao ZW, Jiang XJ. MicroRNA-124-3p inhibits the growth and metastasis of nasopharyngeal carcinoma cells by targeting STAT3. Oncol Rep. 2016;35(3):1385-1394.

14. Hanna RA, Campbell RL, Davies PL. Calcium-bound structure of calpain and its mechanism of inhibition by calpastatin. Nature. 2008; 456(7220):409-412.

15. Storr SJ, Carragher NO, Frame MC, Parr T, Martin SG. The calpain system and cancer. Nat Rev Cancer. 2011;11(5):364-374

16. Zhuang Q, Qian X, Cao Y, Fan M, Xu X, He X. Capn 4 mRNA level is correlated with tumour progression and clinical outcome in clear cell renal cell carcinoma. J Int Med Res. 2014;42(2):282-291.
17. Bai DS, Dai Z, Zhou J, et al. Capn4 overexpression underlies tumor invasion and metastasis after liver transplantation for hepatocellular carcinoma. Hepatology. 2009;49(2):460-470.

18. Zheng PC, Chen X, Zhu HW, et al. Capn4 is a marker of poor clinical outcomes and promotes nasopharyngeal carcinoma metastasis via nuclear factor- $\mathrm{kB}$-induced matrix metalloproteinase 2 expression. Cancer Sci. 2014;105(6):630-638.

19. Lluri G, Langlois GD, Soloway PD, Jaworski DM. Tissue inhibitor of metalloproteinase-2 (TIMP-2) regulates myogenesis and beta1 integrin expression in vitro. Exp Cell Res. 2008;314(1):11-24.

20. Zhao Y, Ling Z, Hao Y, et al. MiR-124 acts as a tumor suppressor by inhibiting the expression of sphingosine kinase 1 and its downstream signaling in head and neck squamous cell carcinoma. Oncotarget. 2017; 8(15):25005-25020.

21. Feng T, Xu D, Tu C, et al. MiR-124 inhibits cell proliferation in breast cancer through downregulation of CDK4. Tumour Biol. 2015;36(8): 5987-5997.

22. Shimada M, Greer PA, McMahon AP, Bouxsein ML, Schipani E. In vivo targeted deletion of calpain small subunit, Capn4, in cells of the osteoblast lineage impairs cell proliferation, differentiation, and bone formation. J Biol Chem. 2008;283(30):21002-21010.

23. Cataldo F, Peche LY, Klaric E, et al. CAPNS1 regulates USP1 stability and maintenance of genome integrity. Mol Cell Biol. 2013; 33(12):2485-2496.

24. Cai JJ, Qi ZX, Hua W, et al. Increased expression of Capn4 is associated with the malignancy of human glioma. CNS Neurosci Ther. 2014; 20(6):521-527.

25. Gu J, Xu FK, Zhao GY, et al. Capn4 promotes non-small cell lung cancer progression via upregulation of matrix metalloproteinase 2 . Med Oncol. 2015;32(3):51.

26. Cai JJ, Qi ZX, Chen LC, Yao Y, Gong Y, Mao Y. miR-124 suppresses the migration and invasion of glioma cells in vitro via Capn4. Oncol Rep. 2016;35(1):284-290.

27. Mao J, Fan S, Ma W, et al. Roles of Wnt/ $\beta$-catenin signaling in the gastric cancer stem cells proliferation and salinomycin treatment. Cell Death Dis. 2014;5:e1039.

28. Chen S, Sun YY, Zhang ZX, Li YH, Xu ZM, Fu WN. Transcriptional suppression of microRNA-27a contributes to laryngeal cancer differentiation via GSK-3 $\beta$-involved Wnt/ $\beta$-catenin pathway. Oncotarget. 2017;8(9):14708-14718.

29. Peng YY, He YH, Chen C, et al. NLRC5 regulates cell proliferation, migration and invasion in hepatocellular carcinoma by targeting the Wnt/ $\beta$-catenin signaling pathway. Cancer Lett. 2016;376(1):10-21.

30. Shang YC, Wang SH, Xiong F, et al. Wnt3a signaling promotes proliferation, myogenic differentiation, and migration of rat bone marrow mesenchymal stem cells 1. Acta Pharmacol Sin. 2007;28(11):1761-1774.

31. Cheng Y, Phoon YP, Lung ML. Inhibition of both physiological and aberrant $\mathrm{Wnt} / \beta$-catenin signaling activities associated with stemness in nasopharyngeal carcinoma. Poster presented at: The 105th Annual Meeting of the American Association for Cancer Research (AACR 2014); April 5-9; 2014; San Diego, CA. abstract no. 1938.

32. Zhang J, Wen X, RenXY, etal. YPEL3 suppresses epithelial-mesenchymal transition and metastasis of nasopharyngeal carcinoma cells through the Wnt/ $\beta$-catenin signaling pathway. J Exp Clin Cancer Res. 2016; 35(1):109.

33. Wang Z, Wang Y, Ren H, Jin Y, Guo Y. ZNRF3 inhibits the invasion and tumorigenesis in nasopharyngeal carcinoma cells by inactivating the Wnt/ $\beta$-catenin pathway. Oncol Res. 2017;25(4):571-577.

34. Yue Q, Zhang Y, Li X, et al. MiR-124 suppresses the chemotactic migration of rat mesenchymal stem cells toward HGF by downregulating Wnt/B-catenin signaling. Eur J Cell Biol. 2016;95(9):342-353.

35. Wang E, Wang D, Li B, et al. Capn4 promotes epithelial-mesenchymal transition in human melanoma cells through activation of the Wnt/ß-catenin pathway. Oncol Rep. 2017;37(1):379-387. 


\section{Publish your work in this journal}

OncoTargets and Therapy is an international, peer-reviewed, open access journal focusing on the pathological basis of all cancers, potential targets for therapy and treatment protocols employed to improve the management of cancer patients. The journal also focuses on the impact of management programs and new therapeutic agents and protocols on

patient perspectives such as quality of life, adherence and satisfaction. The manuscript management system is completely online and includes a very quick and fair peer-review system, which is all easy to use. Visit http://www.dovepress.com/testimonials.php to read real quotes from published authors.

Submit your manuscript here: http://www.dovepress.com/oncotargets-and-therapy-journal 